\title{
Wicksellian Theory of Forest Rotation under Interest Rate Variability
}

\author{
Luis H. R. Alvarez \\ Turku School of Economics and Business Administration \\ and \\ Erkki Koskela \\ University of Helsinki, RUESG and HECER
}

Discussion Paper No. 1

April 2004

ISSN 1795-0562

HECER - Helsinki Center of Economic Research, P.O. Box 17 (Arkadiankatu 7), FI-00014 University of Helsinki, FINLAND, Tel +358-9-191-28780, Fax +358-9-191-28781, E-mail info-hecer@helsinki.fi, Internet www.hecer.fi 


\title{
Wicksellian Theory of Forest Rotation under Interest Rate Variability*
}

\begin{abstract}
We apply the Wicksellian single rotation framework to cover the unexplored case of variable and stochastic interest rate. We provide a mathematical characterization of the two-dimensional optimal stopping problem and show in the presence of amenity valuation that increased interest rate volatility lengthens the optimal rotation period and increases the value of the optimal policy. By modelling the interest rate as a mean reverting process and forest value as a geometric Brownian motion and abstracting from amenity valuation and we present an explicit solution for the problem. Numerical illustrations indicate that interest rate volatility has a significant and nonlinear impact on optimal rotation.
\end{abstract}

JEL Classification: Q23, G31, C61

Keywords: Wicksellian rotation, stochastic interest rates, optimal stopping, free boundary problems.

Luis H. R. Alvarez

Department of Economics,

Quantitative Methods in Management

Turku School of Economics and

Business Administration

Fl-20500 Turku

FINLAND

e-mail: luis.alvarez@tukkk.fi

\section{Erkki Koskela}

Department of Economics

P.O. Box 17 (Arkadiankatu 7)

University of Helsinki

FI-00014 University of Helsinki

FINLAND

e-mail: erkki.koskela@helsinki.fi

\footnotetext{
* The research of Luis H. R. Alvarez has been supported by the Foundation for the Promotion of the Actuarial Profession, Finnish Insurance Society, and the Yrjö Jahnsson Foundation. Erkki Koskela thanks the Research Unit on Economic Structures and Growth (RUESG) in the University of Helsinki funded jointly by the Academy of Finland, University of Helsinki, Bank of Finland, Nokia Group, and the Yrjö Jahnsson Foundation. The authors are grateful to two anonymous referees for helpful comments and to Heikki Ruskeepää for his assistance in the MATHEMATICA ${ }^{\odot}$-calculations. An earlier version of the paper was presented in the 2nd World Congress of Environmental and Resource Economists, Monterey, California, June 24--27, 2002. This paper is forthcoming in Journal of Economic Dynamics and Control.
} 


\section{Introduction}

In forest economics the well-known model by Faustmann 1849 has been the most often used starting point in studies considering the optimal rotation period of forest stands. Under the assumption of constant timber prices, constant total cost of clear-cutting and replanting as well as constant interest rate, perfect capital markets and perfect foresight the model leads to a constant rotation period for an even aged stand, which maximizes the present value of forest stand over an infinite time horizon (see e.g. Clark 1976, Johannsson and Löfgren 1985 and Samuelson 1976). The representative rotation age depends on timber price, total cost of clear-cutting and replanting, nature of forest growth as well as the interest rate.

The basic assumptions and predictions of the Faustmann model do not seem to lie in conformity with empirical evidence (see e.g. Kuuluvainen and Tahvonen 1999). This has led to ongoing research, which has extended the basic Faustmann model under perfect foresight to allow for amenity valuation of timber (see e.g. Hartman 1976), the potential interdependence of forest stands as producers of amenity services (see e.g. Koskela and Ollikainen, 2000, 2001, and Amacher, Koskela, and Ollikainen 2003) as well as imperfect capital markets (see e.g. Tahvonen and Salo and Kuuluvainen 2001). The resulting rotation age has been shown to depend on the properties of amenity valuation function, the nature of stand interdependencies and potential borrowing constraints in the capital markets. In particular, in the latter case most of the basic properties of optimal forest harvesting become different than the ones in the classical Faustmann model.

Finally, the perfect foresight assumption has been relaxed in studies focusing on the implications of stochastic timber prices (see e.g. Brazee and Mendelsohn 1988, Thomson 1992, Plantinga 1998, and Insley 2002), risk of forest fire (see e.g. Reed 1984) and/or stochastic forest growth on optimal rotation age (see e.g. Reed 1993, Miller and Voltaire 1983, Morck and Schwartz and Stangeland 1989, Clarke and Reed 1989, 1990, Willassen 1998 and Alvarez $2003 \mathrm{~b}$ ). The effect of uncertainties on the optimal rotation period depends on the type of uncertainty. In the case of forest fire risk modelled as a Poisson process the rotation age will become shorter due to the higher effective discount rate (see Reed 1984) while in the presence of timber price and/or forest growth risk usually the reverse happens; higher volatility in price or in age-dependent growth will tend to lengthen the rotation period by lowering the effective discount rate. The reason for this finding is that even though increased volatility increases the expected net present value of the harvesting yield, it also raises the value of waiting by increasing the expected net present value of future harvesting opportunities. Since the latter effect dominates the former, higher volatility will unambiguously increase the optimal rotation period (see e.g. Clarke and Reed 1989, Willassen 1998 and Alvarez 2003 b).

This rotation literature has covered several interesting cases and provided useful insights. There is, however, a very important issue, which has not yet been analyzed. To our knowledge in all the research associated with optimal rotation periods of forest stands the assumption of constant interest rate has been sticked to. As we know from empirical research, interest rates fluctuate over time and the implications of this empirical finding for the term structure of interest rates, asset pricing etc. have been one of the major research areas in financial economics (for an up-to-date empirical survey in the field see Cochrane 2001, chapter 20; 
see also Björk 1998, chapter 17 for an extensive treatment of interest rate modelling). If the investment projects would be very liquid ones, then interest rate fluctuations would not necessarily matter very much. In the case of forestry, however, the situation is different. Given the relatively slow growth rate of forests, investing in replanting is a long-term investment project, over which the expected behavior of the interest rate as the opportunity cost will be important. Similarly, since many real investments are productive over a considerably long time period, we are tempted to argue that the variability of interest rates should play a key role in the rational valuation and exercise policies of real irreversible investment opportunities as well. Ingersoll and Ross 1992 have analyzed the effect of interest rate uncertainty on the timing of investment but they model the interest rate process as a martingale (i.e. as a process which has no drift). Alvarez and Koskela 2003 generalizes their findings by allowing for stochastic interest rate of a mean reverting type.

In this paper we analyze the unexplored issue of what is the impact of variable and stochastic interest rate on optimal forest rotation. Since our main emphasis is to consider the impact of a stochastic interest rate on the optimal rotation policy, we first model the underlying interest rate dynamics as a general one factor diffusion process without explicit parametrization of the model. In this way, we plan to establish robust results valid for most well-established one factor interest rate models appearing in the financial literature (cf. Björk 1998 chapter 17, Black and Karasinski 1991, Cox, Ingersoll, and Ross 1980, 1981, 1985, Ingersoll and Ross 1992, Merton 1973, 1975, and Vasiček 1977). We provide a mathematical characterization of the two-dimensional optimal stopping problem and show among others in the presence of amenity valuation that allowing for interest rate uncertainty will increase the optimal rotation period under the natural condition when the value of the optimal policy is convex in terms of the initial interest rate and present plausible conditions under which this holds. We also establish that increased interest rate volatility will increase the value of the optimal policy and move the exercise date further, meaning that the rotation period becomes longer. Finally, modelling interest rate uncertainty as a mean-reverting process and forest value as a geometric Brownian motion, we abstract from amenity valuation and provide an explicit solution for the two-dimensional path-dependent optimal stopping problem. Numerical illustrations indicate that interest rate volatility has a significant nonlinear quantitative importance on the optimal rotation policy.

We proceed as follows: In section 2 we present a framework to study the Wicksellian single rotation problem in the thus far unexplored situation of stochastic interest rate variability in the presence of amenity valuation. Since the problem is more general than the constant discounting case, we first provide a mathematical characterization of the optimal rotation policy and its value, and then state the main results. In section 3 we abstract from amenity valuation and provide a solvable model when we specify interest rate uncertainty as a mean reverting process and forest value as a geometric Brownian motion. Section 4 presents some concluding remarks. 


\section{The Wicksellian Rotation Problem under Interest Rate Uncertainty}

In this section we formulate the Wicksellian rotation problem in the presence of amenity valuation in more general terms than usually by allowing stochastic interest rate variability. We proceed as follows. First we provide a set of sufficient conditions under which the optimal rotation problem admits a unique solution and under which the value of optimal policy can be obtained from an associated boundary value problem subject to standard value matching and smooth fit (or smooth pasting) conditions. Second, we analyze the relationship between increased volatility and the optimal rotation period.

In what follows, we model the stochastic interest rate dynamics as a general one factor diffusion model without explicitly parametrizing the drift of the underlying dynamics. This is because our purpose is to explore the impact of interest rate uncertainty on optimal rotation under very general assumptions in order to be able to establish robust results which would be valid for most well-established one factor interest rate models appearing in the literature of financial economics (cf. Björk 1998 chapter 17, Black and Karasinski 1991, Cox, Ingersoll, and Ross 1980, 1981, 1985, Ingersoll and Ross 1992, Merton 1973, 1975, and Vasiček 1977). In line with these arguments, we assume that the interest rate process $\left\{r_{t} ; t \geq 0\right\}$ is defined on a complete filtered probability space $\left(\Omega, P,\{t\}_{t \geq 0},\right)$ satisfying the usual conditions and that $r_{t}$ is described on $\mathbb{R}_{+}$by the (Itô-) stochastic differential equation

$$
d r_{t}=\alpha\left(r_{t}\right) d t+\sigma\left(r_{t}\right) d W_{t}, \quad r_{0}=r
$$

where $W_{t}$ denotes standard Brownian motion, the drift coefficient $\alpha: \mathbb{R}_{+} \mapsto \mathbb{R}$ is continuously differentiable with a Lipschitz continuous derivative, and the volatility coefficient $\sigma: \mathbb{R}_{+} \mapsto$ $\mathbb{R}_{+}$is a sufficiently smooth mapping for guaranteeing the existence of a solution for (2.1) (at least continuous; cf. Borodin and Salminen 2002, pp. 46-48). In order to avoid interior singularities, we also assume that $\sigma(r)>0$ for all $r \in(0, \infty)$, that $\infty$ is a natural boundary for the diffusion $r_{t}$ (non-explosive paths), and that 0 is either unattainable or exit for $r_{t}$ (cf. Borodin and Salminen 2002, pp. 14-21). It is worth observing that if both boundaries are unattainable and

$$
\int_{0}^{\infty} m^{\prime}(y) d y<\infty
$$

where $m^{\prime}(r)=2 /\left(\sigma^{2}(r) S^{\prime}(r)\right)$ denotes the density of the speed measure $m$ of the diffusion $r_{t}$ and

$$
S^{\prime}(r)=\exp \left(-\int \frac{2 \alpha(r)}{\sigma^{2}(r)} d r\right)
$$

denotes the density of the scale function of the diffusion $r_{t}$, then $r_{t}$ will tend towards a long run steady state distributed according to the stationary distribution with density (cf. Borodin and Salminen 2002, pp. 35-37, see also Merton 1975)

$$
p(r)=\frac{m^{\prime}(r)}{\int_{0}^{\infty} m^{\prime}(y) d y} .
$$


Having presented the dynamics describing the evolution of the interest rate, we now specify the deterministic dynamics for the forest value as follows

$$
d X_{t}=\mu\left(X_{t}\right) d t, \quad X_{0}=x \in \mathbb{R}_{+},
$$

where $\mu: \mathbb{R}_{+} \mapsto \mathbb{R}$ is a known Lipschitz-continuous mapping measuring the growth rate of the forest value. It is now clear that given our assumptions on the underlying dynamics the differential operator associated with the two-dimensional process $\left(X_{t}, r_{t}\right)$ now reads as

$$
\mathcal{A}_{\sigma}=\frac{1}{2} \sigma^{2}(r) \frac{\partial^{2}}{\partial r^{2}}+\mu(x) \frac{\partial}{\partial x}+\alpha(r) \frac{\partial}{\partial r} .
$$

Given the stochastic interest rate dynamics (2.1) and the deterministic forest value dynamics (2.2) we next consider the following Wicksellian stochastic single rotation problem (an optimal stopping problem)

$$
V_{\sigma}(x, r)=\sup _{\tau} E_{(x, r)}\left[\int_{0}^{\tau} e^{-\int_{0}^{s} r_{t} d t} \pi\left(X_{s}\right) d s+e^{-\int_{0}^{\tau} r_{s} d s} g\left(X_{\tau}\right)\right]
$$

where $\tau$ is an arbitrary $\mathcal{F}_{t}$-stopping time, $g: \mathbb{R}_{+} \mapsto \mathbb{R}_{+}$is a continuously differentiable and non-decreasing mapping denoting the payoff accrued from exercising the irreversible harvesting opportunity. In (2.3) the mapping $\pi: \mathbb{R}_{+} \mapsto \mathbb{R}_{+}$measures the monetary flow of returns accrued from leaving the harvesting opportunity unexercised, and it is assumed to be non-negative and continuous in terms of the forest value. Moreover, in order to guarantee the finiteness of the objective, we also assume that both the expected present value of the exercise payoff $g(x)$ and the expected cumulative present value of the flow $\pi(x)$ from the present up to an arbitrarily distant future are bounded for all states. Put formally, we assume that

$$
E_{(x, r)}\left[e^{-\int_{0}^{t} r_{s} d s} g\left(X_{t}\right)\right]<\infty \quad \text { for all }(t, x, r) \in \mathbb{R}_{+}^{3}
$$

and that

$$
E_{(x, r)} \int_{0}^{\infty} e^{-\int_{0}^{s} r_{t} d t} \pi\left(X_{s}\right) d s<\infty \quad \text { for all }(x, r) \in \mathbb{R}_{+}^{2}
$$

The value function is denoted as $V_{\sigma}(x, r)$ in order to emphasize the relationship between volatility and the value of the optimal rotation policy. We can now restate the optimal rotation problem (2.3) by decomposing it into the immediate exercise payoff and the early exercise premium as is indicated by the observation

$$
V_{\sigma}(x, r)=g(x)+F_{\sigma}(x, r),
$$

where

$$
F_{\sigma}(x, r)=\sup _{\tau} E_{(x, r)} \int_{0}^{\tau} e^{-\int_{0}^{t} r_{s} d s}\left[\pi\left(X_{t}\right)+\mu\left(X_{t}\right) g^{\prime}\left(X_{t}\right)-r_{t} g\left(X_{t}\right)\right] d t
$$

denotes the early exercise premium in the presence of interest rate uncertainty. 
Our main objective is to present a characterization of the comparative static properties of the optimal rotation policy and its value as functions of the volatility of the underlying interest rate process. To this end, we assume that the interest rate process $\left\{\hat{r}_{t} ; t \geq 0\right\}$ is described on $\mathbb{R}_{+}$by the (Itô-) stochastic differential equation

$$
d \hat{r}_{t}=\alpha\left(\hat{r}_{t}\right) d t+\hat{\sigma}\left(\hat{r}_{t}\right) d W_{t}, \quad \hat{r}_{0}=r
$$

where $\hat{\sigma}: \mathbb{R}_{+} \mapsto \mathbb{R}_{+}$is again a sufficiently smooth mapping for guaranteeing the existence of a solution for (2.1) (at least continuous; cf. Borodin and Salminen 2002, pp. 46-48) and satisfies the inequality $\hat{\sigma}(r) \geq \sigma(r)$. Put somewhat differently, $\hat{r}_{t}$ can be interpreted as a diffusion evolving at the same rate as $r_{t}$ but subject to greater stochastic fluctuations than $r_{t}$. We emphasize that although in most analyzes the comparison is between different versions (in terms of volatility) of a given underlying interest rate process, we also consider the cases where these processes may be different. In line with our previous notation, we denote as $V_{\hat{\sigma}}(x, r)$ the value of the optimal rotation policy and as $F_{\hat{\sigma}}(x, r)$ the early exercise premium in the presence of the more volatile interest rate dynamics $\hat{r}_{t}$.

Along the lines indicated by previous studies considering the impact of increased volatility on the value of contingent contracts (cf. Alvarez 2001 b, 2003 a, 2003 c, Bergman, Grundy, and Wiener 1996, El Karoui, Jeanblanc-Picqué, and Shreve 1998, Hobson 1998, and Janson and Tysk 2003) the convexity of the value function plays a key role in the determination of the sign of the relationship between increased volatility and the value of the optimal rotation policy. Hence, it is important to ask: under what conditions the value $V_{\sigma}(x, r)$ of the optimal policy under interest rate uncertainty is a convex function of the initial interest rate. Before establishing our main characterization of the sign of the relationship between volatility and the optimal rotation policy, we present the following result characterizing both the convexity of the expected revenues and their dependence on the volatility of the underlying interest rate process.

Lemma 2.1. Assume that either $\sigma(r)=\sigma r$ and that $\alpha(r)$ is concave or that the interest rate model is affine (i.e. that both $\alpha(r)$ and $\sigma^{2}(r)$ are linear). Under these assumptions, the total expected present value of the future harvesting yield in the presence of amenity valuation

$$
G_{\sigma}(t, x, r)=E_{(x, r)}\left[\int_{0}^{t} e^{-\int_{0}^{s} r_{t} d t} \pi\left(X_{s}\right) d s+e^{-\int_{0}^{t} r_{s} d s} g\left(X_{t}\right)\right]
$$

is a decreasing and convex function of the initial interest rate. Moreover, increased volatility of the underlying interest rate process increases its value.

Proof. See Appendix A.

Lemma 2.1 states a set of conditions under which the total expected present value of the future harvesting yield in the presence of amenity valuation is a decreasing and convex mapping of the initial interest rate. Moreover, it also establishes that given its assumptions, higher interest rate volatility unambiguously increases the value of the future harvesting yield in the presence of amenity valuation. These findings are essentially based on the observation that given the assumptions of Lemma 2.1 the initial values of zero coupon bonds maturing 
at arbitrary future dates are decreasing and convex mappings of the initial interest rate and increased volatility increases their value for all maturities.

Our main result characterizing the sign of the relationship between increased volatility and the optimal rotation policy and its value is now summarized in the following.

Theorem 2.2. Assume that the conditions of Lemma 2.1 are satisfied. Then, $V_{\hat{\sigma}}(x, r) \geq$ $V_{\sigma}(x, r)$ and $F_{\hat{\sigma}}(x, r) \geq F_{\sigma}(x, r)$ for all $(x, r) \in \mathbb{R}_{+}^{2}$, and $\left\{(x, r) \in \mathbb{R}_{+}^{2}: V_{\sigma}(x, r)>g(x)\right\} \subset$ $\left\{(x, r) \in \mathbb{R}_{+}^{2}: V_{\hat{\sigma}}(x, r)>g(x)\right\}$ so that increased volatility increases both the value and the early exercise premium of the irreversible policy and, therefore, prolongs the optimal rotation period by expanding the continuation region where harvesting is suboptimal.

Proof. See Appendix B.

Theorem 2.2 demonstrates that given the conditions of our Lemma 2.1, increased interest rate volatility unambiguously increases the value of the harvesting opportunity in the presence of amenity valuation and, consequently, postpones the optimal harvesting decision by expanding the continuation region where harvesting is suboptimal. This observation is essentially based on the fact that increased interest rate volatility increases both the expected present value of the exercise payoff and the expected cumulative present value of amenities while leaving the exercise payoff unchanged. This means that the required exercise premium increases which, in turn, postpones the rational exercise of the harvesting opportunity (cf. Dixit and Pindyck 1994, chapter 5).

\section{A Solvable Single Rotation Model}

In this section we provide an explicit solution for the two-dimensional path-dependent optimal stopping problem and illustrate our findings also numerically. More specifically, we abstract from amenity valuation and model the stochastic interest rate dynamics as an explicitly parametrized mean-reverting process (which lies in conformity with empirical evidence, see e.g. Cochrane 2001, chapter 19) and forest value in a simpler way as a geometric Brownian motion.

Consider the following (path-dependent) optimal rotation problem

$$
V(x, r)=\sup _{\tau} \mathbf{E}_{(x, r)}\left[e^{-\int_{0}^{\tau} r_{s} d s} X_{\tau}\right]
$$

where the underlying processes $\left(X_{t}, r_{t}\right)$ evolve according to the dynamics described by the following stochastic differential equations

$$
d r_{t}=\alpha r_{t}\left(1-\gamma r_{t}\right) d t+\sigma r_{t} d W_{t}, \quad r_{0}=r
$$

and

$$
d X_{t}=\mu X_{t} d t+\beta X_{t} d \hat{W}_{t}, \quad X_{0}=x
$$


where $\alpha, \beta, \sigma, \gamma, \mu \in \mathbb{R}_{+}$are known exogenously given constants and $W_{t}$ and $\hat{W}_{t}$ are potentially correlated Wiener processes (under the objective probability measure $\mathbb{P}$ ) with a known correlation coefficient $\rho \in[-1,1]$.

Having characterized the underlying stochastic dynamics and the considered Wicksellian optimal rotation problem, we are now in position to state the following.

Lemma 3.1. The Wicksellian two-dimensional path-dependent single rotation problem (3.1) can be re-expressed as a path-independent optimal stopping problem

$$
V(x, r)=x r^{-\frac{1}{\alpha \gamma}} \sup _{\tau} \mathbf{E}_{r}\left[e^{-\theta \tau} \hat{r}_{\tau}^{\frac{1}{\alpha \gamma}}\right]
$$

where

$$
\theta=\frac{1}{\gamma}-\mu-\frac{\sigma^{2}}{2 \alpha \gamma}\left(1+\frac{1}{\alpha \gamma}\right)+\frac{\sigma \beta \rho}{\alpha \gamma}
$$

can be interpreted as a "risk-adjusted" discount rate and the interest rate $\tilde{r}_{t}$ evolves according to the dynamics described by the following stochastic differential equation

$$
d \tilde{r}_{t}=\left(\alpha+\beta \sigma \rho-\frac{\sigma^{2}}{\alpha \gamma}-\alpha \gamma \tilde{r}_{t}\right) \tilde{r}_{t} d t+\sigma \tilde{r}_{t} d W_{t}, \quad \tilde{r}_{0}=r .
$$

Proof. See Appendix C.

It is worth emphasizing that the findings of Lemma 3.1 are important since they demonstrate how the original path-dependent single rotation problem can be transformed into an ordinary path-independent optimal stopping problem of a linear diffusion. Our main result in this section is now summarized in the following

Theorem 3.2. Assume that the risk-adjusted discount rate is positive (i.e. $\theta>0$ ) guaranteeing the finiteness of the value of optimal policy. Then the value of the single rotation problem (3.1) reads as

$$
V(x, r)=x r^{-\frac{1}{\alpha \gamma}} \psi(r) \sup _{y \geq r}\left[\frac{y^{\frac{1}{\alpha \gamma}}}{\psi(y)}\right]= \begin{cases}x, & r \geq r^{*} \\ x\left(\frac{r^{*}}{r}\right)^{\frac{1}{\alpha \gamma}} \frac{\psi(r)}{\psi\left(r^{*}\right)}, & r<r^{*}\end{cases}
$$

where

$$
\psi(r)=r^{\eta} M\left(\eta, 2 \eta+\frac{2 a}{\sigma^{2}}, \frac{2 \alpha \gamma}{\sigma^{2}} r\right),
$$

$\eta=\frac{1}{2}-\frac{a}{\sigma^{2}}+\sqrt{\left(\frac{1}{2}-\frac{a}{\sigma^{2}}\right)^{2}+\frac{2 \theta}{\sigma^{2}}}>0, a=\alpha+\beta \sigma \rho-\frac{\sigma^{2}}{\alpha \gamma}$, and $M$ denotes the standard confluent hypergeometric function (see e. g. Dixit and Pindyck 1994, p. 163). Moreover, the optimal interest rate exercise threshold $r^{*}$ is the unique root of the ordinary first order condition $\psi\left(r^{*}\right)=\alpha \gamma r^{*} \psi^{\prime}\left(r^{*}\right)$. Especially, $r^{*}>\mu$ for all $\sigma>0$ and $r^{*}=\mu$ when $\sigma=0$.

Proof. $L(r)=\sup _{\tau} \mathbf{E}_{r}\left[e^{-\theta \tau} \tilde{r}_{\tau}^{\frac{1}{\alpha \gamma}}\right]$ is an ordinary path-independent optimal stopping problem of a linear diffusion and, therefore, can be solved by relying on ordinary variational inequalities. The alleged result is then a direct implication of Theorem 3 in Alvarez 2001 a. 
Theorem 3.2 interestingly demonstrates that the path-dependent optimal rotation problem (3.4) is explicitly solvable whenever the absence of speculative bubbles condition $\theta>0$, which guarantees the finiteness of the value of the optimal rotation policy, is satisfied. It is worth noticing that in the absence of uncertainty the condition $\theta>0$ can be simply expressed as $1 / \gamma>\mu$ meaning that the steady-state interest rate exceeds the growth rate of forest value. On the other hand, under uncertainty about the interest rate and forest value the absence of speculative bubbles condition $\theta>0$ can also be re-expressed as

$$
\frac{1}{\gamma}>\mu+\frac{\sigma^{2}}{2 \alpha \gamma}\left(1+\frac{1}{\alpha \gamma}\right)-\frac{\sigma \beta \rho}{\alpha \gamma}
$$

Thus, we naturally find that the condition $\theta>0$ is strengthened by the presence of uncertainty whenever the correlation $\rho$ between the two driving Brownian motions is non-positive and is weakened whenever the correlation is positive. Moreover, and importantly, higher volatility increases the required exercise premium and thus prolongs the expected length of the optimal rotation period.

Remark: It is worth noticing that since

$$
d X_{t}^{b}=\left(b \mu+\frac{1}{2} \beta^{2} b(b-1)\right) X_{t}^{b} d t+b \beta X_{t}^{b} d \hat{W}_{t}
$$

the result of Theorem 3.2 can be applied for solving the associated optimal stopping problem

$$
H(x, r)=\sup _{\tau} \mathbf{E}_{(x, r)}\left[e^{-\int_{0}^{\tau} r_{s} d s} X_{\tau}^{b}\right]
$$

where $b \in \mathbb{R}$ is a known parameter measuring the curvature of the mapping $x^{b}$. As is clear from Theorem 3.2, in that case we find that provided that the absence of speculative bubbles condition $\tilde{\theta}=\frac{1}{\gamma}-b \mu-\frac{1}{2} \beta^{2} b(b-1)-\frac{\sigma^{2}}{2 \alpha \gamma}\left(1+\frac{1}{\alpha \gamma}\right)+\frac{\sigma b \beta \rho}{\alpha \gamma}>0$ is satisfied the value of the stopping problem (3.6) reads as

$$
H(x, r)=x^{b} r^{-\frac{1}{\alpha \gamma}} \tilde{\psi}(r) \sup _{y \geq r}\left[\frac{y^{\frac{1}{\alpha \gamma}}}{\tilde{\psi}(y)}\right]= \begin{cases}x^{b}, & r \geq \tilde{r} \\ x^{b}\left(\frac{\tilde{r}}{r}\right)^{\frac{1}{\alpha \gamma}} \frac{\tilde{\psi}(r)}{\tilde{\psi}(\tilde{r})}, & r<\tilde{r}\end{cases}
$$

where

$$
\tilde{\psi}(r)=r^{\tilde{\eta}} M\left(\tilde{\eta}, 2 \tilde{\eta}+\frac{2 \tilde{a}}{\sigma^{2}}, \frac{2 \alpha \gamma}{\sigma^{2}} r\right)
$$

$\tilde{\eta}=\frac{1}{2}-\frac{\tilde{a}}{\sigma^{2}}+\sqrt{\left(\frac{1}{2}-\frac{\tilde{a}}{\sigma^{2}}\right)^{2}+\frac{2 \tilde{\theta}}{\sigma^{2}}}>0$, and $\tilde{a}=\alpha+b \beta \sigma \rho-\frac{\sigma^{2}}{\alpha \gamma}$. Now, the optimal exercise threshold $\tilde{r}$ is the unique root of the ordinary first order condition $\tilde{\psi}(\tilde{r})=\alpha \gamma \tilde{r} \tilde{\psi}^{\prime}(\tilde{r})$.

Finally, we characterize the quantitative significance and more precise role of the volatility coefficient $\sigma$ by numerical illustrations. Assume that $\gamma=25, \alpha=0.07, \rho=0$ and $\mu=0.01$ (implying that for $\theta>0$ the upper bound under which the absence of speculative bubbles 
condition is satisfied is $\left.\sigma^{*}=0.2585\right)$. Then, the optimal threshold $r^{*}$ and required exercise premium $r^{*}-\mu$ as a function of the underlying volatility coefficient are

\begin{tabular}{|c|c|c|c|c|}
\hline$\sigma$ & 0.1 & 0.2 & 0.25 & 0.258 \\
\hline$r^{*}$ & $1.1 \%$ & $1.58 \%$ & $2.77 \%$ & $4.37 \%$ \\
$r^{*}-\mu$ & $0.1 \%$ & $0.58 \%$ & $1.77 \%$ & $3.37 \%$ \\
\hline
\end{tabular}

\section{Table 1}

According to the findings presented in Table 1 the required exercise premium increases from $0.1 \%$ to $3.37 \%$ as volatility increases from 0.1 to 0.258 . In order to illustrate our results in the negative correlation case, we assume that $\gamma=25, \alpha=0.07, \rho=-0.5$ and $\mu=0.01$ (implying that now $\sigma^{*}=0.2286$ ). Then the optimal threshold and required exercise premium as a function of the underlying volatility coefficient are

\begin{tabular}{|c|c|c|c|}
\hline$\sigma$ & 0.1 & 0.2 & 0.22 \\
\hline$r^{*}$ & $1.1 \%$ & $1.86 \%$ & $2.62 \%$ \\
$r^{*}-\mu$ & $0.1 \%$ & $0.86 \%$ & $1.62 \%$ \\
\hline
\end{tabular}

\section{Table 2}

Thus, we find that the required exercise premium increases from $0.1 \%$ to $1.62 \%$ as volatility increases from 0.1 to 0.22 . According to these numerical illustrations, higher interest rate volatility has a very big effect on the required exercise premium, thus implying a significantly longer optimal rotation period. In fact, numerical calculations seem to indicate that the expected length of the optimal rotation period increases at a faster rate than interest rate volatility so that the relationship between the expected length of the optimal rotation period and interest rate volatility seems to be nonlinearly positive even in the presence of risk neutrality. Consequently, even a small change in the volatility of the underlying interest rate dynamics may result into a disproportionate impact on the expected duration of a rotation cycle. Thus, our findings demonstrate that destabilizing polices will result in the mean into longer rotation periods (this question has been raised in a different context, cf. Dixit and Pindyck, 1994, p. 14).

\section{Conclusions}

There is currently an extensive literature about the determination of optimal forest rotation under various circumstances when amenity valuation of forest stands matters, when capital markets are imperfect so that landowners might be subject to borrowing constraints or when there is uncertainty about timber prices and/or forest growth or about risk of forest fire. Undoubtedly this literature has provided useful insights about the potential determinants of optimal forest rotation. There is, however, an important issue, which has not yet been analyzed. To our knowledge all the literature makes a simplifying but in the forestry case an unrealistic assumption that the interest rate is constant. Clearly the irreversible harvesting 
decision of forest stands is a decision subject to a relatively long time horizon. Hence, given the relatively slow growth rate of forests, thinking about harvesting and investing in replanting is a long-term investment project over which the behavior of interest rates as the opportunity cost should matter a lot.

In this paper we have used the Wicksellian single rotation framework to extend the existing studies to cover the unexplored case of variable and stochastic interest rate in the presence of amenity valuation. Since the problem is more general than the constant discounting case, we first provided a mathematical characterization of the optimal rotation policy as a two-dimensional path-dependent optimal stopping problem.

From an economic point of view we have established several new findings. First, we have demonstrated in the presence of amenity valuation that allowing for interest rate uncertainty will increase the optimal rotation period under the condition that the value of the optimal policy is convex in terms of interest rate. Second, under the plausible assumptions that the diffusion term in the (Itô-) stochastic differential equation for the interest rate is sufficiently smooth as a function of the interest rate and the drift term is concave function of the interest rate, higher interest rate volatility will increase the value of waiting and prolong the optimal rotation period in the absence of amenity valuation. Third, modelling interest rate uncertainty as a mean-reverting process and forest value as a geometric Brownian motion, we have provided an explicit solution for the two-dimensional path-dependent optimal stopping problem. Numerical illustrations indicate that interest rate volatility has a significant quantitative importance on the optimal rotation policy. In particular, the expected length of the optimal rotation period will increase proportionally more than interest rate volatility even in the presence of risk neutrality.

Whether our conclusions remain valid in the Faustmann's ongoing rotation problem is an open question beyond the scope of the present study. However, given the close connection of impulse control problems and optimal stopping theory (impulse control problems can be viewed as sequential stopping problems; cf. Alvarez 2003 b), we are tempted to conjecture that most probably our conclusions would remain valid with only minor modifications in the ongoing rotation case as well at least for some class of amenity valuation functions. Of course, the verification of this claim is still an open and challenging problem left for future research. 


\section{References}

[1] Alvarez, L. H. R. Reward functionals, salvage values, and optimal stopping, 2001 a, Mathematical Methods of Operations Research, 54, 315-337.

[2] Alvarez, L. H. R. On the form and risk-sensitivity of zero coupon bonds for a class of interest rate models, 2001 b, Insurance Mathematics and Economics, 28, 83-90.

[3] Alvarez, L. H. R. On the convexity and risk-sensitivity of the price of american interest rate derivatives, 2003 a, SIAM Journal on Applied Mathematics, 63, 923-936.

[4] Alvarez, L. H. R. Stochastic forest growth and Faustmann's formula, 2003 b, SIAM Journal on Control and Optimization, to appear.

[5] Alvarez, L. H. R. On the properties of $r$-excessive mappings for a class of diffusions, 2003 c, Annals of Applied Probability, to appear.

[6] Alvarez, L. H. R. and Koskela, E. Irreversible investment under interest rate variability, 2003, Journal of Business, to appear.

[7] Amacher, G. S., Koskela, E., and Ollikainen, M. Forest rotations and stand interdependency: Ownership structure and timing of decisions, 2003, University of Helsinki, Department of Economics, DP 584 (to appear in Natural Resource Modeling).

[8] Bergman, Y., Grundy, B., and Wiener, Z. General properties of option prices, 1996, Journal of Finance, 51, 1573-1610.

[9] Black, F. and Karasinski, P. Bond and option pricing when short rates are lognormal, 1991, Financial Analysts Journal, 47, 52-59.

[10] Björk, T. Arbitrage Theory In Continuous Time, 1998, Oxford University Press, Somerset.

[11] Borodin, A. and Salminen, P. Handbook on Brownian motion - Facts and formulae, 2nd edition, 2002, Birkhauser, Basel.

[12] Brazee, R. and Meldelsohn, R. Timber harvesting with fluctuating prices, 1988, Forest Science, 34, 359-372.

[13] Clark, C. W. Mathematical Bioeconomics: The optimal management of renewable resources, 1976, Wiley, New York.

[14] Clarke, H. R. and Reed, W. J. The tree-cutting problem in a stochastic environment, 1989, Journal of Economic Dynamics and Control, 13, 569-595.

[15] Clarke, H.R. and Reed, W.J Harvest decisions and asset valuations for biological resources exhibiting size-dependent stochastic growth, 1990, International Economic Review, 31, 147-169. 
[16] Cochrane, J.H. Asset Pricing, 2001, Princeton University Press.

[17] Cox, J. C., Ingersoll, J. E., and Ross, S. A. An analysis of variable rate loan contracts, 1980, Journal of Finance, 35, 389-403.

[18] Cox, J. C., Ingersoll, J. E., and Ross, S. A. A re-examination of traditional expectation hypotheses about the term structure of interest rates, 1981, Journal of Finance, 36, 769799.

[19] Cox, J. C., Ingersoll, J. E., and Ross, S. A. A theory of the term structure of interest rates, 1985, Econometrica, 53, 385-407.

[20] Dixit, A. K. and Pindyck, R. S. Investment under Uncertainty, 1994, Princeton University Press, Princeton.

[21] Duffie, D. Security Markets: Stochastic Models, 1988, Academic Press, San Diego.

[22] El Karoui, N., Jeanblanc-Picqué, M., and Shreve, S. E. Robustness of the Black-Scholes Formula, 1998, Mathematical Finance, 8, 93 - 126.

[23] Faustmann, M. Berechnung des Wertes welchen Waldboden sowie noch nicht haubare Holzbestände für die Waldwirtschaft besitzen, 1849, Allgemeine Forst- unde Jagd-Zeitung, 15, 441-455.

[24] Hartman, R. The harvesting decision when a standing forest has value, 1976, Economic Inquiry, 14, 52-58.

[25] Hobson, D. G. Volatility mis-specification, option pricing and super-replication via coupling, 1998, Annals of Applied Probability, vol 8, 193 - 205.

[26] Ingersoll, J. E., Jr. and Ross, S. A. Waiting to invest: Investment and uncertainty, 1992, Journal of Business, 65, $1-29$.

[27] Insley, M. A real options approach to the valuation of a forestry investment, 2002, Journal of Environmental Economics and Management, 44, 471-492.

[28] Jansson, S. and Tysk, J. Volatility time and properties of option prices, 2003, Annals of Applied Probability, 13, 890-913.

[29] Johansson, P. O. and Löfgren, K. G. The economics of forestry and natural resources, 1985, Basil Blackwell, Oxford.

[30] Koskela, E. and Ollikainen, M. Forest taxation and rotation age under private amenity valuation: new results, 2001, Journal of Environmental Economics and Management, 42, 374-384.

[31] Koskela, E. and Ollikainen, M. Optimal private and public harvesting under spatial and temporal interdependence, 2001, Forest Science, 47, 484-496. 
[32] Kuuluvainen, J. and Tahvonen, O. Testing the forest rotation model: evidence from panel data, 1999, Forest Science, 45, 539-551.

[33] Merton, R. C. A rational theory of option pricing, 1973, Bell Journal of Economics and Management Science, 41, 141-183.

[34] Merton, R. C. An asymptotic theory of growth under uncertainty, 1975, Review of Economic Studies, 42, 375-393.

[35] Miller, R. A. and Voltaire, K. A stochastic analysis of the three paradigm, 1983, Journal of Economic Dynamics and Control, 6, 371-386.

[36] Morck, R. and Schwartz, E. and Stangeland, D. The valuation of forestry resources under stochastic prices and inventories, 1989, Journal of Financial and Quantitative Analysis, 24,473-487.

[37] Øksendal, B. Stochastic differential equations: An introduction with applications, (Sixth Edition) 2003, Springer, Berlin.

[38] Plantinga, A. J. The optimal timber rotation: an option value approach, 1998, Forest Science, 44, 192-202.

[39] Protter, P. Stochastic integration and differential equations, 1990, Springer, New York.

[40] Reed, W. J. The effects of the risk of fire on the optimal rotation of a forest, 1984, Journal of Environmental Economics and Management, 11, 180-190.

[41] Reed, W. J. The decision to conserve or harvest old-growth forest, 1993, Ecological Economics, 8, 45-69.

[42] Samuelson, P. A. Economics of forestry in an evolving society, 1976, Economic Inquiry, 14, 466-492.

[43] Tahvonen, O. and Salo, S. and Kuuluvainen, J. Optimal forest rotation and land values under a borrowing constraint, 2001, Journal of Economic Dynamics and Control, 25, $1595-1627$.

[44] Thomson, T. A. Optimal forest rotation when stumpage prices follow a diffusion process, 1992, Land Economics, 68, 329-342.

[45] Vasiček, O. An equilibrium characterization of the term structure, 1977, Journal of Financial Economics, 5, 177-188.

[46] Willassen, Y. The stochastic rotation problem: A generalization of Faustmann's formula to stochastic forest growth, 1998, Journal of Economic Dynamics and Control, 22, 573596. 


\section{A Proof of Lemma 2.1}

Proof. Consider first the case where $\alpha(r)$ is concave and $\sigma(r)=\bar{\sigma} r$, where $\bar{\sigma}>0$ is a known exogenously given constant. We follow the proof of Theorem 2 in Alvarez $2001 \mathrm{~b}$. Denote now as $r_{t}(i), t \geq 0$, the solution of the stochastic differential equation (2.1) subject to the initial condition $r_{0}=i \in \mathbb{R}_{+}$. Given our smoothness assumptions $r_{t}(i)$ can be expressed in the (Itô-) form

$$
r_{t}(i)=i+\int_{0}^{t} \alpha\left(r_{s}(i)\right) d s+\int_{0}^{t} \bar{\sigma} r_{s}(i) d W_{s}
$$

where $r_{t}(i)$ constitutes a continuously differentiable mapping of $i$ (this is based on the flow nature of the solution of a stochastic differential equation; cf. Protter 1990, Theorem V. 38 and 39). Define now the process $\left\{Y_{t} ; t \geq 0\right\}$ as $Y_{t}=\partial r_{t}(i) / \partial i$. It is then well-known that (cf. Protter 1990, Theorem V. 39)

$$
Y_{t}=1+\int_{0}^{t} \alpha^{\prime}\left(r_{s}(i)\right) Y_{s} d s+\int_{0}^{t} \bar{\sigma} Y_{s} d W_{s}
$$

Applying Itô's theorem to the mapping $y \mapsto \ln y$ then implies that the solution of the stochastic differential equation (A.2) can be expressed as

$$
Y_{t}=\frac{\partial r_{t}(i)}{\partial i}=\exp \left(\int_{0}^{t} \alpha^{\prime}\left(r_{s}(i)\right) d s\right) Z_{t}
$$

where, given our assumptions, the process $\left\{Z_{t} ; t \geq 0\right\}$ defined as $Z_{t}=\exp \left(\bar{\sigma} W_{t}-\bar{\sigma}^{2} t / 2\right)$ is a positive martingale starting at date 0 from 1 for any possible $i \in \mathbb{R}_{+}$. The concavity of the drift $\alpha(r)$ then implies that $\alpha^{\prime}(r)$ is non-increasing in $r$ and that $\alpha^{\prime}\left(r_{s}(\rho)\right) \leq \alpha^{\prime}\left(r_{s}(i)\right)$ for all $\rho \geq i$ and $s \in[0, t]$. Consequently, we find that $\partial r_{t}(i) / \partial i$ is non-increasing in $i$, proving the alleged concavity of the solution $r_{t}(i)$ as a function of $i$. Since a decreasing and convex transformation of an increasing and concave mapping is decreasing and convex, we observe that the discount factor $e^{-\int_{0}^{t} r_{s} d s}$ is a decreasing and convex function of the initial interest rate. Hence, the mapping

$$
G_{\sigma}(t, x, r)=E_{(x, r)}\left[\int_{0}^{t} e^{-\int_{0}^{s} r_{t} d t} \pi\left(X_{s}\right) d s+e^{-\int_{0}^{t} r_{s} d s} g\left(X_{t}\right)\right]
$$

is a decreasing and convex function of the initial interest rate $r$ as well. It remains to establish that increased volatility increases the value of $G_{\sigma}(t, x, r)$. To accomplish this task, we first observe that the functional $G_{\sigma}(t, x, r)$ can be re-expressed as

$$
G_{\sigma}(t, x, r)=\int_{0}^{t} p^{s}(r) \pi\left(X_{s}\right) d s+p^{t}(r) g\left(X_{t}\right)
$$

where

$$
p^{t}(r)=E_{r}\left[e^{-\int_{0}^{s} r_{t} d t}\right]
$$


denotes the value at time 0 of a zero coupon bond maturing at $t$. It is now clear form our results above that $p^{t}(r)$ is a decreasing and convex function of the initial interest rate $r$. Define now for all $t \in[0, T]$ the bounded and twice continuously differentiable mapping $P^{T}:[t, T] \times \mathbb{R}_{+} \mapsto[0,1]$ as (cf. Björk 1998, chapter 16)

$$
P^{T}(t, r)=E_{r}\left[e^{-\int_{t}^{T} r_{s} d s}\right]
$$

and observe that $P^{T}(T, r)=1$ and that $P^{T}(0, r)=p^{T}(r)$. Since $P^{T}(t, r)$ satisfies the boundary value problem (by the Feynman-Kač-formula; see, for example, Duffie 1988, p. 226 and Øksendal 2003, p. 143)

$$
\begin{aligned}
\frac{\partial P^{T}}{\partial t}(t, r)+\alpha(r) \frac{\partial P^{T}}{\partial r}(t, r)+\frac{1}{2} \sigma^{2}(r) \frac{\partial^{2} P^{T}}{\partial r^{2}}(t, r)-r P^{T}(t, r) & =0 \\
P^{T}(T, r) & =1
\end{aligned}
$$

we find by applying Itô's theorem to the mapping $P^{T}(t, r)$ that

$$
\begin{aligned}
E_{r}\left[e^{-\int_{t}^{T} \hat{r}_{s} d s} P^{T}\left(T, \hat{r}_{T}\right)\right] & =P^{T}(t, r)+E_{r} \int_{t}^{T} e^{-\int_{t}^{s} \hat{r}_{y} d y} \frac{1}{2}\left(\hat{\sigma}^{2}\left(\hat{r}_{s}\right)-\sigma^{2}\left(\hat{r}_{s}\right)\right) P_{r r}^{T}\left(s, \hat{r}_{s}\right) d s \\
& \geq P^{T}(t, r) .
\end{aligned}
$$

Since $P^{T}\left(T, \hat{r}_{T}\right)=1$ we observe that $\hat{P}^{T}(t, r) \geq P^{T}(t, r)$, where

$$
\hat{P}^{T}(t, r)=E_{r}\left[e^{-\int_{t}^{T} \hat{r}_{s} d s}\right] .
$$

Hence, we find that increased volatility increases the current (date 0) value of the zero coupon bonds $p^{t}(r)$ and, therefore, that $G_{\sigma}(t, x, r) \leq G_{\hat{\sigma}}(t, x, r)$.

It remains to consider the impact of higher interest volatility in the presence of an affine term structure. It is well-known that in that case the price of zero coupon bonds read as $p^{T}(t, r)=e^{A(t, T)-B(t, T) r}$ (cf. Björk 1998) implying the monotonicity and convexity of the price of zero coupon bonds. The alleged positivity of the sign of the relationship between interest rate volatility and the expected cumulative present value of the harvesting yield now follows from the analysis above.

\section{B Proof of Theorem 2.2}

Proof. As was established in Lemma 2.1, the discount factor $e^{-\int_{0}^{t} r_{s} d s}$ is decreasing and convex as a function of the initial interest rate $r$. Given this observation, define now the increasing sequence $\left\{V_{n}(x, r, y)\right\}_{n \in \mathbb{N}}$ iteratively as

$$
\begin{aligned}
V_{0}(x, r, y) & =\sup _{t \geq 0} E_{(x, r, y)}\left[e^{-\int_{0}^{t} r_{s} d s} g\left(X_{t}\right)+Y_{t}\right] \\
V_{n+1}(x, r, y) & =\sup _{t \geq 0} E_{(x, r, y)}\left[e^{-\int_{0}^{t} r_{s} d s} V_{n}\left(X_{t}, r_{t}, Y_{t}\right)\right],
\end{aligned}
$$


where the process $Y_{t}$ evolves according to the dynamics described by the differential equation (cf. Øksendal 2003, pp. 222-223)

$$
d Y_{t}=e^{-\int_{0}^{t} r_{s} d s} \pi\left(X_{t}\right) d t, \quad Y_{0}=y
$$

It is known that the sequence of mappings $V_{n}(x, r, y)$ converges towards the value function $\bar{V}(x, r, y)$ satisfying the condition $\bar{V}(x, r, 0)=V_{\sigma}(x, r)$ (cf. Øksendal 2003, p. 210). It is again clear from Lemma 2.1 that, given the assumed positivity of the monetary flow of returns $\pi(x)$, $Y_{t}$ is a decreasing and convex function of the initial interest rate $r$. Similarly, the positivity of the exercise payoff $g(x)$ implies that the expected present value of the exercise payoff is a decreasing and convex function of the initial interest rate $r$ as well. Since the sum of decreasing and convex functions is itself a decreasing and convex function and the maximum of a convex function is convex, we find that $V_{0}(x, r, y)$ is convex and decreasing as a function of the initial interest rate $r$. Consequently, all elements in the sequence $\left\{V_{n}(x, r, y)\right\}_{n \in \mathbb{N}}$ are decreasing and convex as functions of $r$. Since $V_{n}(x, r, 0) \uparrow V_{\sigma}(x, r)$ as $n \rightarrow \infty$ (cf. Øksendal 2003, p. 210) we find that for all $\lambda \in[0,1]$ and $r, \rho \in \mathbb{R}_{+}$we have that

$$
\lambda V_{\sigma}(x, r)+(1-\lambda) V_{\sigma}(x, \rho) \geq \lambda V_{n}(x, r, 0)+(1-\lambda) V_{n}(x, \rho, 0) \geq V_{n}(x, \lambda r+(1-\lambda) \rho, 0) .
$$

Letting $n \rightarrow \infty$ and invoking monotone convergence then implies that $\lambda V_{\sigma}(x, r)+(1-$ ג) $V_{\sigma}(x, \rho) \geq V_{\sigma}(x, \lambda r+(1-\lambda) \rho)$ proving the convexity of $V_{\sigma}(x, r)$. The alleged monotonicity of the value function can be established in a completely analogous way.

It remains to establish that higher volatility increases the value and, therefore, postpones optimal rotation by expanding the continuation region where exercising the harvesting opportunity is suboptimal. To see that this is indeed the case, we first observe that Lemma 2.1 implies that $V_{0}(x, r, y) \leq \hat{V}_{0}(x, r, y)$ where

$$
\hat{V}_{0}(x, r, y)=\sup _{t \geq 0} E_{(x, r, y)}\left[e^{-\int_{0}^{t} \hat{r}_{s} d s} g\left(X_{t}\right)+\hat{Y}_{t}\right]
$$

and

$$
d \hat{Y}_{t}=e^{-\int_{0}^{t} \hat{r}_{s} d s} \pi\left(X_{t}\right) d t, \quad \hat{Y}_{0}=y .
$$

Consequently, we find that $\hat{V}_{n}(x, r, y) \geq V_{n}(x, r, y)$ for all $n \in \mathbb{N}$, where

$$
\hat{V}_{n+1}(x, r, y)=\sup _{t \geq 0} E_{(x, r, y)}\left[e^{-\int_{0}^{t} \hat{r}_{s} d s} \hat{V}_{n}\left(X_{t}, \hat{r}_{t}, \hat{Y}_{t}\right)\right]
$$

Combining this observation with the monotonicity of the sequence $\left\{\hat{V}_{n}(x, r, y)\right\}_{n \in \mathbb{N}}$, letting $n \uparrow \infty$, and invoking monotone convergence finally implies that $V_{\hat{\sigma}}(x, r) \geq V_{\sigma}(x, r)$. The inequality $F_{\hat{\sigma}}(x, r) \geq F_{\sigma}(x, r)$ now follows from the definition of the early exercise premium. Finally, if $(x, r) \in C_{\sigma}=\left\{(x, r) \in \mathbb{R}_{+}^{2}: V_{\sigma}(x, r)>g(x)\right\}$, then $V_{\sigma}(x, r) \geq V_{\sigma}(x, r)>g(x)$ proving that $(x, r) \in C_{\hat{\sigma}}=\left\{(x, r) \in \mathbb{R}_{+}^{2}: V_{\hat{\sigma}}(x, r)>g(x)\right\}$ as well and, therefore, that $C_{\sigma} \subset C_{\hat{\sigma}}$. 


\section{Proof of Lemma 3.1}

Proof. It is well-known that the forest value process can in the case of our study be expressed as

$$
X_{t}=x \exp \left(\left(\mu-\beta^{2} / 2\right) t+\beta \hat{W}_{t}\right)
$$

Moreover, applying Itô's theorem to the mapping $r \mapsto \ln r$ yields

$$
\ln \left(r_{t} / r\right)=\left(\alpha-\frac{1}{2} \sigma^{2}\right) t-\alpha \gamma \int_{0}^{t} r_{s} d s+\sigma W_{t}
$$

which in turn implies

$$
e^{-\int_{0}^{t} r_{s} d s}=\left(\frac{r_{t}}{r}\right)^{\frac{1}{\alpha \gamma}} e^{\frac{\left(\sigma^{2}-2 \alpha\right) t}{2 \alpha \gamma}-\frac{\sigma W_{t}}{\alpha \gamma}}
$$

Hence, we observe that the present value of the forest stand reads as

$$
e^{-\int_{0}^{t} r_{s} d s} X_{t}=x\left(\frac{r_{t}}{r}\right)^{\frac{1}{\alpha \gamma}} e^{-\left(\frac{1}{\gamma}-\mu-\frac{\sigma^{2}}{2 \alpha \gamma}\left(1+\frac{1}{\alpha \gamma}\right)+\frac{\sigma \beta \rho}{\alpha \gamma}\right) t} M_{t}
$$

where

$$
M_{t}=e^{\beta \hat{W}_{t}-\frac{\sigma}{\alpha \gamma} W_{t}+\left(\frac{\sigma \beta \rho}{\alpha \gamma}-\frac{1}{2} \beta^{2}-\frac{\sigma^{2}}{2 \alpha^{2} \gamma^{2}}\right) t}
$$

is a positive exponential $\mathcal{F}_{t}$-martingale. Consequently, we find that the path-dependent Wicksellian optimal rotation problem (3.1) can be re-expressed as an ordinary path-independent optimal stopping problem

$$
V(x, r)=x r^{-\frac{1}{\alpha \gamma}} \sup _{\tau} \mathbf{E}_{(x, r)}\left[e^{-\theta \tau} r_{\tau}^{\frac{1}{\alpha \gamma}} M_{\tau}\right]
$$

where

$$
\theta=\frac{1}{\gamma}-\mu-\frac{\sigma^{2}}{2 \alpha \gamma}\left(1+\frac{1}{\alpha \gamma}\right)+\frac{\sigma \beta \rho}{\alpha \gamma}
$$

can be interpreted as a "risk-adjusted" discount rate. Defining the equivalent measure $\mathbb{Q}$ as $\frac{d \mathbb{Q}}{d \mathbb{P}}=M_{t}$ then implies that we can now re-express (C.1) as

$$
V(x, r)=x r^{-\frac{1}{\alpha \gamma}} \sup _{\tau} \mathbf{E}_{(x, r)}^{\mathbb{Q}}\left[e^{-\theta \tau} r_{\tau}^{\frac{1}{\alpha \gamma}}\right] .
$$

However, given the strong uniqueness of a solution for the stochastic differential equation

$$
d r_{t}=\left(\alpha+\beta \sigma \rho-\frac{\sigma^{2}}{\alpha \gamma}-\alpha \gamma r_{t}\right) r_{t} d t+\sigma r_{t} d \tilde{W}_{t}, \quad r_{0}=r
$$

where $\tilde{W}_{t}$ is a standard Brownian motion under the equivalent measure $\mathbb{Q}$, we finally find that the rotation problem (3.1) can be rewritten in the path-independent form (3.4) defined under the objective measure $\mathbb{P}$. 